\title{
Pattern formations of 2D Rayleigh-Bénard convection with no-slip boundary conditions for the velocity at the critical length scales
}

\author{
Taylan Sengul ${ }^{\mathrm{a}}$, Jie Shen ${ }^{\mathrm{b}}$ and Shouhong Wang ${ }^{\mathrm{k}+}$
}

\section{Communicated by R. Showalter}

We study the Rayleigh-Bénard convection in a 2D rectangular domain with no-slip boundary conditions for the velocity. The main mathematical challenge is due to the no-slip boundary conditions, because the separation of variables for the linear eigenvalue problem, which works in the free-slip case, is no longer possible. It is well known that as the Rayleigh number crosses a critical threshold $R_{c}$, the system bifurcates to an attractor, which is an $(m-1)$-dimensional sphere, where $\boldsymbol{m}$ is the number of eigenvalues, which cross zero as $R$ crosses $R_{c}$. The main objective of this article is to derive a full classification of the structure of this bifurcated attractor when $m=2$. More precisely, we rigorously prove that when $m=2$, the bifurcated attractor is homeomorphic to a one-dimensional circle consisting of exactly four or eight steady states and their connecting heteroclinic orbits. In addition, we show that the mixed modes can be stable steady states for small Prandtl numbers. Copyright $\odot 2014$ John Wiley \& Sons, Ltd.

Keywords: Bénard convection; dynamic transition; pattern formation

\section{Introduction}

The Rayleigh-Bénard convection problem is one of the fundamental problems in the physics of fluids. The basic phenomena of the Rayleigh-Bénard convection in horizontally extended systems are widely known. The influence of the side walls, although not studied as throughly as the horizontally extended case, is of practical importance for engineering applications.

In this paper, we study the Rayleigh-Bénard convection in a 2D rectangular domain with no-slip boundary conditions for the velocity. This problem is also closely related to the problem of infinite channel with rectangular cross section, which has been studied by DaviesJones [1], Luijkx and Platten [2], and Kato and Fujimura [3], among others.

The linear aspects of the problem we consider in this paper have been studied by Lee et al. [4], Mizushima [5], and Gelfgat [6]. In these papers, the critical Rayleigh number and the structure of the critical eigenmodes have been studied for small aspect ratio containers.

From dynamical transition and pattern formation point of view, Ma and Wang $[7,8]$ proved that under some general boundary conditions, the problem always undergoes a dynamic transition to an attractor $\Sigma_{R}$ as the Rayleigh number $R$ crosses the first critical Rayleigh number $R_{c}$. They also proved that the bifurcated attractor is homological to $S^{m-1}$, where $m$ is the number of critical eigenmodes.

In the $2 \mathrm{D}$ setting that we consider, $m$ is either 1 or 2 , and the latter case can only happen at the critical length scales where two modes with wave numbers $k$ and $k+1$ become critical simultaneously. When $m=1$, the structure of $\Sigma_{R}$ is trivial, which is merely a disjoint union of two attracting steady states. Thus, our task in this paper is to classify the structure of the attractor when $m=2$. This has been studied recently in [9] for the 3D Rayleigh-Bénard problem where the boundaries were assumed to be free slip for the velocity and the wave numbers of the critical modes were assumed to be equal.

The main mathematical challenge in this paper is due to the no-slip boundary conditions because the separation of variables for the linear eigenvalue problem, which works in the free-slip case, is not possible anymore. To overcome this difficulty, the main approach for our study is to combine rigorous analysis and numerical computation using spectral method.

\footnotetext{
a Department of Mathematics, Yeditepe University, 34750 Istanbul, Turkey

${ }^{b}$ Department of Mathematics, Purdue University, West Lafayette, IN, USA

${ }^{c}$ Department of Mathematics, Indiana University, Bloomington, IN, USA

* Correspondence to: Shouhong Wang, Department of Mathematics, Indiana University, Bloomington, IN, USA.

†E-mail: showang@indiana.edu
} 
As we know, spectral methods have long been used to address the hydrodynamic instability problems. In fact, in his seminal work [10], Orszag studied the classical Orr-Sommerfeld linear instability problem using a Chebyshev-tau method. In this paper, to treat the linear eigenvalue problem, we employ a Legendre-Galerkin method where compact combinations of Legendre polynomials, also called generalized Jacobi polynomials, satisfying all the boundary conditions are used as trial functions. The main advantage of Legendre-Galerkin method is that the resulting matrices are sparse, which allows a very efficient and accurate solution of the linearized problem; see also Hill and Straughan [11] and Gheorghiu and Dragomirescu [12].

Once the eigenpairs of the linear problem are identified, the transition analysis is carried out by reducing the infinite dimensional system to the center manifold in the first two critical eigendirections. The coefficients of this reduced system are calculated numerically. Our main results are described later.

We first classify the eigenmodes into four classes according to their parities using the symmetry of the problem. Then, we numerically show that the first two unstable modes are always parity class 1 or 2 . Then, we study the transition near the critical length scales where two eigenvalues become positive simultaneously. Next, we rigorously prove that the local attractor at small supercritical Rayleigh numbers is in fact homeomorphic to the circle, which has four or eight steady states with half of them as stable points and the rest as saddle points. The critical eigenmodes are always bifurcated steady states on the attractor, and when the attractor has eight steady states, the mixed modes, which are superpositions of the critical eigenmodes, are also bifurcated.

Second, let $\beta_{1}$ and $\beta_{2}$ denote the two largest eigenvalues of the linearized problem. We find that a small neighborhood of $\beta_{1}=$ $\beta_{2}=0$ in the $\beta_{1}-\beta_{2}$ plane can be separated into several sectors with different asymptotical structures. In particular, we find that there is a critical Prandtl number $\operatorname{Pr}_{c}$ for the first two critical length scales $L=1.5702$ and $L=2.6611$, such that for $\operatorname{Pr}<\operatorname{Pr}$, there is a sector in this plane for which mixed modes are stable fixed points of the attractor. For $\operatorname{Pr}>\operatorname{Pr}_{c}$, the mixed modes are never stable, and instead, there is a sector in this plane in which both of the critical eigenmodes coexist as stable steady states. In this case, the initial conditions determine which one of these eigenmodes will be realized. The critical Prandtl number $\operatorname{Pr}_{c}$ is around 0.14 for the first critical length scale $L=1.5702$ and around 0.05 for the second critical length scale $L=2.6611$. For higher critical length scales, we found that mixed modes are never stable points of the attractor.

Third, recently, Ma and Wang have developed the dynamic transition theory to study transition and bifurcation problems in nonlinear sciences; see [13]. This paper is a first attempt to combine this theory with the numerical tools of the spectral methods to study the detailed structure of the transition and pattern formation.

The paper is organized as follows. In Section 2, the governing equations and the functional setting of the problem are discussed. In Section 3, linear eigenvalue problem is studied. Section 4 states the main theorem. Section 5 is devoted to the proof of the main theorem. In Section 6, we demonstrate a method to compute the coefficients of the reduced system. And the last section discusses the results obtained by our analysis.

\section{Governing equations and the functional setting}

Two-dimensional thermal convection with no-slip, perfectly conducting boundaries can be modeled by the Boussinesq equations. The governing equations on the rectangular domain $\Omega=(0, L) \times(0,1) \in \mathbb{R}^{2}$ read as

$$
\begin{aligned}
\frac{\partial \mathbf{u}}{\partial t}+(\mathbf{u} \cdot \nabla) \mathbf{u} & =\operatorname{Pr}(-\nabla p+\Delta \mathbf{u})+\sqrt{R} \sqrt{\operatorname{Pr}} \theta \mathbf{k} \\
\frac{\partial \theta}{\partial t}+(\mathbf{u} \cdot \nabla) \theta & =\sqrt{R} \sqrt{\operatorname{Pr}} w+\Delta \theta \\
\nabla \cdot \mathbf{u} & =0 .
\end{aligned}
$$

Here, $\mathbf{u}=(u, w)$ is the velocity field, $\theta$ is the temperature field, and $p$ is the pressure field. These fields represent a perturbation around the motionless state with a linear temperature profile. The dimensionless numbers are the Prandtl number Pr and the Rayleigh number $R$, which is also the control parameter. $\mathbf{k}$ represents the unit vector in the $z$-direction.

The equations (1) are supplemented with no-slip boundary conditions for the velocity and perfectly conducting boundary conditions for the temperature.

$$
\mathbf{u}=\theta=0, \quad \text { on } \partial \Omega \text {. }
$$

For the functional setting, we define the relevant function spaces:

$$
\begin{aligned}
H & =\left\{(\mathbf{u}, \theta) \in L^{2}\left(\Omega, \mathbb{R}^{3}\right): \nabla \cdot \mathbf{u}=0,\left.\mathbf{u} \cdot n\right|_{\partial \Omega}=0\right\} \\
H_{1} & =\left\{(\mathbf{u}, \theta) \in H^{2}\left(\Omega, \mathbb{R}^{3}\right): \nabla \cdot \mathbf{u}=0,\left.\mathbf{u}\right|_{\partial \Omega}=0,\left.\theta\right|_{\partial \Omega}=0\right\} .
\end{aligned}
$$

For $\phi=(\mathbf{u}, \theta)$, let $G: H_{1} \rightarrow H$ and $L_{R}: H_{1} \rightarrow H$ be defined by

$$
\begin{aligned}
L_{R} \phi & =(\mathcal{P}(\operatorname{Pr} \Delta \mathbf{u}+\sqrt{R} \sqrt{\operatorname{Pr}} \theta \mathbf{k}), \sqrt{R} \sqrt{\operatorname{Pr}} w+\Delta \theta) \\
G(\phi) & =-(\mathcal{P}(\mathbf{u} \cdot \nabla) \mathbf{u},(\mathbf{u} \cdot \nabla) \theta))
\end{aligned}
$$

with $\mathcal{P}$ denoting the Leray projection onto the divergence-free vectors. 
The equations (1)-(2) supplemented with initial conditions can be put into the following abstract ordinary differential equation:

$$
\frac{d \phi}{d t}=L_{R} \phi+G(\phi), \quad \phi(0)=\phi_{0}
$$

For results concerning the existence and uniqueness of solutions of (5), we refer to Foias et al. [14].

Finally, for $\phi_{i}=\left(\mathbf{u}_{i}, \theta_{i}\right), \mathbf{u}_{i}=\left(u_{i}, w_{i}\right), i=1,2,3$, we define the following trilinear forms.

$$
\begin{aligned}
G\left(\phi_{1}, \phi_{2}, \phi_{3}\right) & =-\int_{\Omega}\left(\mathbf{u}_{1} \cdot \nabla\right) \mathbf{u}_{2} \cdot \mathbf{u}_{3} d x d z-\int_{\Omega}\left(\mathbf{u}_{1} \cdot \nabla\right) \theta_{2} \cdot \theta_{3} d x d z \\
G_{s}\left(\phi_{1}, \phi_{2}, \phi_{3}\right) & =G\left(\phi_{1}, \phi_{2}, \phi_{3}\right)+G\left(\phi_{2}, \phi_{1}, \phi_{3}\right) .
\end{aligned}
$$

\section{Linear analysis}

We first study the eigenvalue problem $L_{R} \phi=\beta \phi$, which reads as

$$
\begin{aligned}
\operatorname{Pr}\left(\Delta u-\frac{\partial p}{\partial x}\right) & =\beta u, \\
\operatorname{Pr}\left(\Delta w-\frac{\partial p}{\partial z}\right)+\sqrt{R} \sqrt{\operatorname{Pr}} \theta & =\beta w, \\
\Delta \theta+\sqrt{R} \sqrt{\operatorname{Pr}} w & =\beta \theta, \\
\operatorname{div} \mathbf{u} & =0, \\
u & =\theta=0, \quad \text { at } \partial \Omega .
\end{aligned}
$$

In the following, we list some of the properties of this eigenvalue problem.

1. The linear operator $L_{R}$ is symmetric. Hence, the eigenvalues $\beta_{i}$ are real, and the eigenfunctions $\phi_{i}$ are orthogonal with respect to $L^{2}$-inner product. Moreover, there is a sequence

$$
0<R_{1} \leq R_{2} \leq \cdots
$$

such that $\beta_{i}\left(R_{i}\right)=0 . R_{i}$ is found by setting $\beta=0$ in (7). In this case, the problem becomes an eigenvalue problem with $\sqrt{R}$ as the eigenvalue.

2. We have

$$
\beta_{i}(R) \underset{<}{\gtrless} 0 \text { if } R \underset{<}{\gtrless} R_{i}
$$

which can be seen by computing the derivative of $\beta_{i}$ with respect to $R$ at $R=R_{i}$.

$$
\left.\frac{d \beta_{i}}{d R}\right|_{R=R_{i}}=\frac{1}{\sqrt{R_{i}}} \frac{\sqrt{\operatorname{Pr}} \int_{\Omega} \theta_{i} w_{i} d x d z}{\int_{\Omega}\left(u_{i}^{2}+w_{i}^{2}+\theta_{i}^{2}\right) d x d z},
$$

where $\left(u_{i}, w_{i}, \theta_{i}\right)$ is the $i^{t \text { th }}$ eigenfunction. Also at $R=R_{i}$, by the third equation in (7), $w_{i}=-R_{i}^{-1 / 2} \operatorname{Pr}^{-1 / 2} \Delta \theta_{i}$ as $\beta_{i}\left(R_{i}\right)=0$. Plugging these into (8) and integrating by parts, we see that

$$
\left.\frac{d \beta_{i}}{d R}\right|_{R=R_{i}}=\frac{\int_{\Omega}\left|\nabla \theta_{i}\right|^{2} d x d z}{R_{i} \int_{\Omega}\left(\left|u_{i}\right|^{2}+\left|w_{i}\right|^{2}+\left|\theta_{i}\right|^{2}\right) d x d z}>0
$$

3. We denote the critical Rayleigh number $R_{c}=R_{1}$. That is,

$$
\begin{aligned}
& \beta_{i}(R)\left\{\begin{array}{l}
<0 \text { if } R<R_{c \prime} \\
=0 \text { if } R=R_{c \prime} \quad i=1, \ldots, m \\
>0 \text { if } R>R_{c} .
\end{array}\right. \\
& \beta_{i}\left(R_{c}\right)<0, \quad i>m .
\end{aligned}
$$

$m$ in (9) does not depend on the Prandtl number Pr but only on $L$. To see this, one makes the change of variable $\theta=\sqrt{\operatorname{Pr}} \theta^{\prime}$ so that the solution of (7) for the eigenvalue of $\beta=0$ is independent of Pr. By simplicity of the first eigenvalue (see Theorem 3.7 in the study of Ma and Wang [15]), for almost every value of $L$ except a discrete set of values, $m$ in (9) is 1 . 
Table I. Possible parity classes of the eigenfunctions of the linear operator.

\begin{tabular}{|cccc|}
\hline Class 1 & Class 2 & Class 3 & Class 4 \\
\hline$\psi(e, e), \theta(o, e)$ & $\psi(o, e), \theta(e, e)$ & $\psi(e, o), \theta(o, o)$ & $\psi(o, o), \theta(e, o)$ \\
\hline
\end{tabular}

Introducing the stream function $\psi_{z}=u, \psi_{x}=-w$, we can eliminate the pressure $p$ from the linear eigenvalue problem (7).

$$
\begin{aligned}
\operatorname{Pr} \Delta^{2} \psi-\sqrt{R} \sqrt{\operatorname{Pr}} \theta_{x} & =\beta(R) \Delta \psi, \\
-\sqrt{R} \sqrt{\operatorname{Pr}} \psi_{x}+\Delta \theta & =\beta(R) \theta, \\
\psi=\frac{\partial \psi}{\partial n}=\theta & =0 \text { on } \partial \Omega .
\end{aligned}
$$

The linear equations (7) satisfy several discrete symmetries, which may be found from the known non-trivial groups of continuous Lie symmetries of the field equations (1); see Hydon [16] and Marques et al. [17]. However, for the problem we consider, it can be easily verified that the linear equations have reflection symmetries about the horizontal and vertical mid-planes of the domain. Thus, we can classify the solutions of the linear problem into four classes with different parities as defined in Table I where, for example, $\psi(0, e)$ means that $\psi$ is odd in the $x$-direction and even in the $z$-direction.

We will employ a Legendre-Galerkin method (cf. Shen [18]; Shen et al. [19]) to solve the linear eigenvalue problem (10). For this, first, we transform the domain with the change of variable

$$
(x, z) \in(0, L) \times(0,1) \rightarrow\left(x^{\prime}, z^{\prime}\right)=\left(\frac{2 x}{L}-1,2 z-1\right) \in(-1,1)^{2}
$$

For notational simplicity, we will omit the primes and write $(x, z) \in(-1,1)^{2}$. The approximate solutions $\left(\psi^{N}, \theta^{N}\right)$ of $(10)$ will be sought in the finite dimensional space, which is the span of

$$
\left\{\left(e_{j}(x) e_{k}(z), f_{l}(x) f_{m}(z)\right) \mid j, I=0, \ldots, N_{x}-1, k, m=0, \ldots, N_{z}-1\right\} \text {, }
$$

where $e_{j}$ and $f_{j}$ are generalized Jacobi polynomials (cf. Guo et al. [20]; Shen et al. [19]), which satisfy the boundary conditions

$$
e_{i}( \pm 1)=D e_{i}( \pm 1)=f_{i}( \pm 1)=0 \text {. }
$$

Here, $D$ denotes the derivative. The polynomials $e_{i}$ and $f_{i}$ are defined as in chapter 6 of Shen et al. [19],

$$
\begin{gathered}
f_{i}(z)=L_{i}(z)-L_{i+2}(z), \\
e_{i}(z)=\frac{L_{i}(z)-\frac{4 i+10}{2 i+7} L_{i+2}(z)+\frac{2 i+3}{2 i+7} L_{i+4}(z)}{(2 i+3)(4 i+10)^{1 / 2}},
\end{gathered}
$$

where $L_{i}$ is the $i^{\text {th }}$ Legendre polynomial. The coefficient of $e_{i}$ guarantees that $\left(D^{2} e_{i}, D^{2} e_{j}\right)=\delta_{i j}$.

We write the approximate solutions of equation (10) with coefficients to be determined by

$$
\psi^{N}=\sum_{j=0}^{N_{x}-1} \sum_{k=0}^{N_{z}-1} \tilde{\psi}_{j k}^{N} e_{j}(x) e_{k}(z), \quad \theta^{N}=\sum_{j=0}^{N_{x}-1} \sum_{k=0}^{N_{z}-1} \tilde{\theta}_{j k}^{N} f_{j}(x) f_{k}(z)
$$

Here, $N=2 N_{x} N_{z}$ denotes the total degrees of freedom.

Let us define for $i, j=0 \ldots, m-1$,

$$
\begin{array}{ll}
\left(A_{1}^{m}\right)_{i j}=\left(D^{2} e_{i}, D^{2} e_{j}\right)=\delta_{i j}, & \left(A_{2}^{m}\right)_{i j}=\left(D^{2} e_{i}, e_{j}\right)=-\left(D e_{j}, D e_{i}\right), \\
\left(A_{3}^{m}\right)_{i j}=\left(e_{i}, e_{j}\right), & \left(A_{4}^{m}\right)_{i j}=\left(e_{i}, f_{j}\right), \\
\left(A_{5}^{m}\right)_{i j}=\left(D^{2} f_{i}, f_{j}\right), & \left(A_{6}^{m}\right)_{i j}=\left(f_{i}, f_{j}\right), \\
\left(A_{7}^{m}\right)_{i j}=\left(D f_{i}, e_{j}\right), &
\end{array}
$$

and for $j=0, \ldots, N_{x}-1, k=0, \ldots, N_{z}-1$,

$$
\tilde{\psi}^{N}=\left\{\tilde{\psi}_{j k}^{N}\right\}, \tilde{\theta}^{N}=\left\{\tilde{\theta}_{j k}^{N}\right\} .
$$


By using the following property of the Legendre polynomials

$$
(2 i+3) L_{i+1}=D\left(L_{i+2}-L_{i}\right)
$$

it is easy to see that

$$
D e_{i}(z)=\frac{L_{i+3}-L_{i+1}}{\sqrt{4 i+10}}, D^{2} e_{i}(z)=\sqrt{\frac{2 i+5}{2}} L_{i+2}(z), D f_{i}=-(2 i+3) L_{i+1} .
$$

By (15), it is easy to determine the elements of the matrices $A_{k}$ by the orthogonality of the Legendre polynomials. In particular, the matrices $A_{1}^{m}, \ldots A_{7}^{m}$ are banded, and except for $A_{4}^{m}$ and $A_{7}^{m}$, they are symmetric.

Putting (13) into (10), multiplying the resulting equations by $e_{m}(x) e_{n}(z)$ and $f_{m}(x) f_{n}(z)$, respectively, and integrating over $-1 \leq x \leq 1$, $-1 \leq z \leq 1$ to obtain

$$
B^{N} \bar{x}^{N}-\sqrt{R} C^{N} \bar{x}^{N}=\beta^{N}(R) D^{N} \bar{x}^{N}
$$

Here,

$$
\begin{aligned}
B^{N}=\left[\begin{array}{cc}
\operatorname{Pr} X_{1} & 0 \\
0 & X_{3}
\end{array}\right]_{N \times N}{ }^{\prime} \quad C^{N}=\left[\begin{array}{cc}
0 & \sqrt{\operatorname{Pr}} X_{2} \\
-\sqrt{\operatorname{Pr}} X_{2}^{T} & 0
\end{array}\right]_{N \times N}{ }^{\prime} \\
D^{N}=\left[\begin{array}{cc}
X_{4} & 0 \\
0 & X_{5}
\end{array}\right]_{N \times N}, \quad \bar{x}^{N}=\left[\begin{array}{l}
\operatorname{vec}\left(\tilde{\psi}^{N}\right) \\
\operatorname{vec}\left(\tilde{\theta}^{N}\right)
\end{array}\right]_{N \times 1}{ }^{\prime}
\end{aligned}
$$

where

$$
\begin{aligned}
& X_{1}=\frac{2^{4}}{L^{4}} A_{3}^{N_{z}} \otimes A_{1}^{N_{x}}+\frac{2^{5}}{L^{2}} A_{2}^{N_{z}} \otimes A_{2}^{N_{x}}+2^{4} A_{1}^{N_{z}} \otimes A_{3}^{N_{x}}, \\
& X_{2}=\frac{2}{L} A_{4}^{N_{z}} \otimes\left(A_{7}^{N_{x}}\right)^{T}, \quad X_{3}=\frac{2^{2}}{L^{2}} A_{6}^{N_{z}} \otimes A_{5}^{N_{x}}+2^{2} A_{5}^{N_{z}} \otimes A_{6}^{N_{x}}, \\
& X_{4}=\frac{2^{2}}{L^{2}} A_{3}^{N_{z}} \otimes A_{2}^{N_{x}}+2^{2} A_{2}^{N_{z}} \otimes A_{3}^{N_{x}}, \quad X_{5}=A_{6}^{N_{z}} \otimes A_{6}^{N_{x}} .
\end{aligned}
$$

In (17) and (18), we use the following notations. For an $m \times k$ matrix $M, \operatorname{vec}(M)$ is the $m k \times 1$ column vector obtained by concatenating the columns $M_{i}$ of $M$, that is,

$$
\operatorname{vec}\left(\left[\begin{array}{llll}
M_{1} & M_{2} & \cdots & M_{k}
\end{array}\right]\right)=\left[\begin{array}{llll}
M_{1}^{T} & M_{2}^{T} \cdots M_{k}^{T}
\end{array}\right]^{T} \text {. }
$$

0 stands for the zero matrix. If $A$ is an $m \times n$ matrix and $B$ is $p \times q$, then $A \otimes B=\left\{a_{i j} B\right\}$, the Kronecker product of $A$ and $B$, is an $m p \times n q$ matrix defined as

$$
A \otimes B=\left[\begin{array}{ccc}
a_{11} B & \ldots & a_{1 n} B \\
\vdots & \ddots & \vdots \\
a_{m 1} B & \ldots & a_{m n} B
\end{array}\right] .
$$

To obtain (16), we used the following properties of the Kronecker product.

- If $A, B$, and $X$ are three matrices such that $A X B$ is defined, then

$$
\operatorname{vec}(A X B)=\left(B^{T} \otimes A\right) \operatorname{vec}(X)
$$

- $(A \otimes B)^{T}=A^{T} \otimes B^{T}$.

We note that the matrices $B^{N}, C^{N}$, and $D^{N}$ in (16) are sparse, $B^{N}$ and $D^{N}$ are symmetric, and $C^{N}$ is skew-symmetric. From our linear analysis, we find the following results.

- Our numerical analysis suggest that $N_{x}=6+2 k \approx 6+2 L$ and $N_{z}=8$ is enough to resolve the critical Rayleigh number and the first critical mode, which has $k$ rolls in its stream function. We have checked that increasing $N_{x}$ and $N_{z}$ by two only modifies the fourth or fifth significant digit of the result.

- In Figure 1, the first critical mode is shown for the length scales $L=1, \ldots, 4$. Note that the first critical stream function and the temperature distribution have always even parity in the $z$-direction while their $x$-parity alternates between odd and even as the length scale increases. As observed by Mizushima [5], we also verify the existence of the Moffatt vortices on the corners of the domain, which are due to corner singularities as shown in Figure 2.

- For $L<21$, we observed that $m$ in (9) is either 1 or 2 . Moreover, $m=2$ only at the critical length scales, which are given in Table II. The results found are in agreement with those in the study of Mizushima [5] and Lee et al. [4].

- The marginal stability curves of the first few critical eigenvalues are given in Figure 3 . The figure demonstrates that the parities of the first two critical modes can only be of parity class 1 or 2 as given in Table I. 

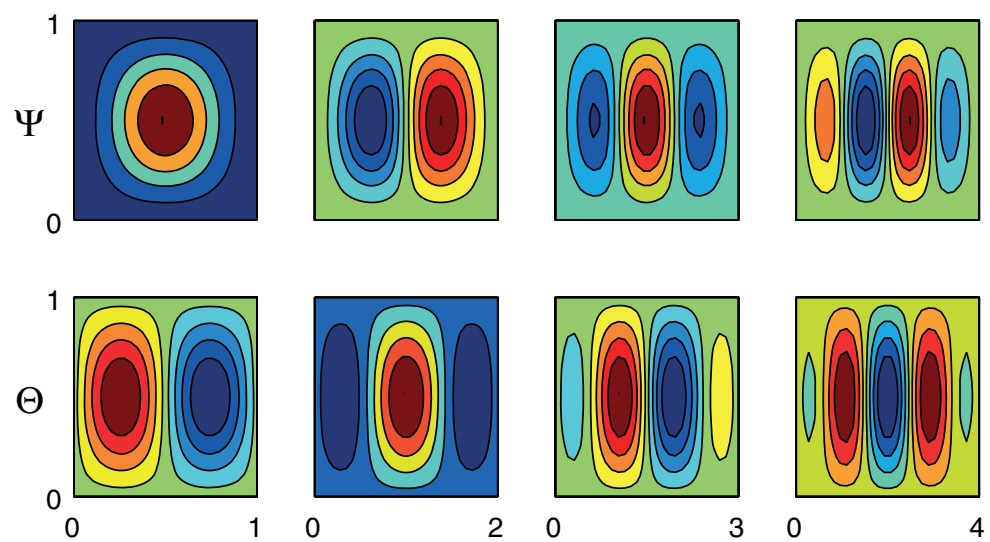

Figure 1. $\psi$ (on top) and $\theta$ (on bottom) of the first critical mode for $L=1, \ldots, 4$.
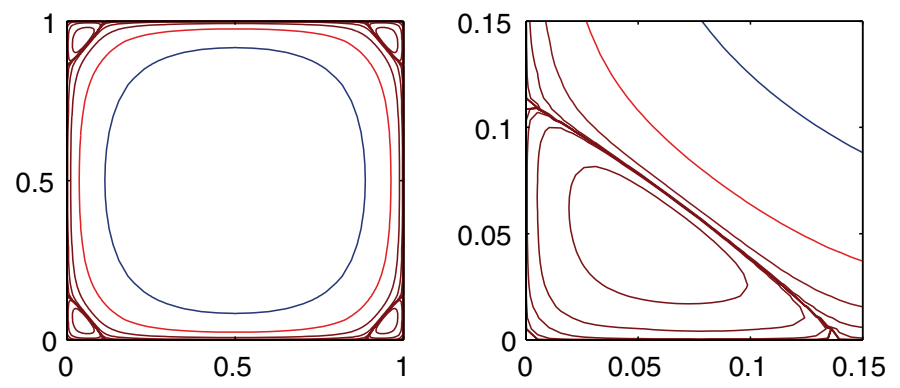

Figure 2. The left figure shows the plot of the first critical stream function for $L=1$ (the top left plot in Figure 1). The right figure shows the corner details of the left figure.

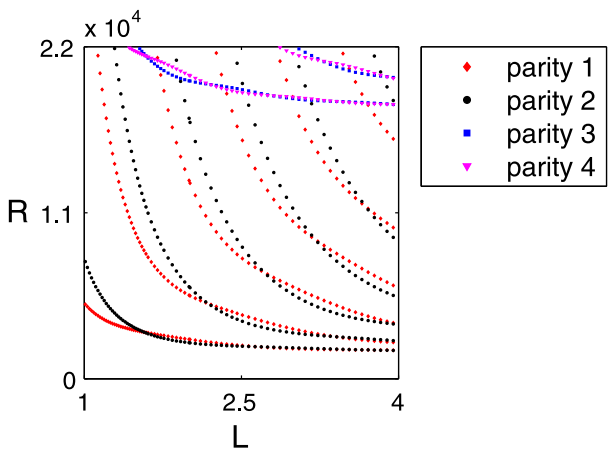

Figure 3. The marginal stability curves of the first few eigenvalues with eigenfunctions of different parity classes.

Also it is seen in these figures that there is a repulsion of the eigenvalues. Namely, the neutral stability curves of the same parity type do not intersect each other. Such a repulsion does not occur for free-slip boundary conditions. This repulsion arises from a structural instability of the transform of matrices into a Jordan canonical form, and a detailed analysis can be found in the study of Mizushima and Nakamura [21].

\section{Main theorem}

Let $m$ be the number of modes, which become critical as the first Rayleigh number $R_{c}$ is crossed as given by (9). Ma and Wang [7,8] proved that under some general boundary conditions, the problem has an attractor $\Sigma_{R}$, which bifurcates from $\left(0, R_{c}\right)$ as $R$ crosses $R_{c}$. They also proved that the dimension of the bifurcated attractor is $m-1 \leq \operatorname{dim}\left(\Sigma_{R}\right) \leq m$. When $m=1$, the structure of $\Sigma_{R}$ is trivial, which is merely a disjoint union of two attracting steady states.

As stated before, in our problem, $m$ is either 1 or 2 . And the latter case can only happen at a critical length scale $L_{c}$ where two eigenmodes with consecutive wave numbers become critical.

Numerically, it turns out that the critical Rayleigh numbers for modes with parity 3 or 4 are much greater than those for modes with parity 1 or 2 . This can be seen from Figure 3. 


\begin{tabular}{|lllrrrrrr|}
\hline \multicolumn{7}{|l|}{ Table II. At $L=L_{c}$, two modes become unstable. } \\
$L_{c}$ & $k$ & \multicolumn{1}{c|}{$R_{c}$} & $N_{x}$ & \multicolumn{1}{c|}{$L_{c}$} & \multicolumn{1}{c|}{$k$} & \multicolumn{1}{c|}{$R_{c}$} & $N_{x}$ \\
\hline 1.5702 & 1 & 3086.6554 & 8 & 6.7711 & 6 & 1764.3754 & 18 \\
2.6611 & 2 & 2113.776 & 10 & 8.7992 & 8 & 1740.9174 & 22 \\
3.7048 & 3 & 1906.3395 & 12 & 10.8229 & 10 & 1729.5398 & 26 \\
4.7329 & 4 & 1826.4099 & 14 & 15.8738 & 15 & 1717.805 & 36 \\
5.7541 & 5 & 1786.8833 & 16 & 20.9197 & 20 & 1713.5226 & 46 \\
\hline
\end{tabular}

One of the modes has $k$, and the other one has $k+1$ rolls in $x$-direction in their stream functions. The critical Rayleigh number at this length scale is $R_{c} . N_{x}$ and $N_{z}$ are the number of polynomials used in the $x$ and $z$ directions, respectively, with $N_{z}=8$ fixed.

We will assume the following.

Consider a solution $\phi$ of (5)

$$
\left\{\begin{array}{l}
\text { 1. }\left(\beta_{1}, \phi_{1}\right),\left(\beta_{2}, \phi_{2}\right) \text { are the first two critical eigenpairs. } \\
\text { 2. } \phi_{1} \text { has wave number } k, \phi_{2} \text { has wave number } k+1 \\
\text { where } k \text { is a positive integer. } \\
\text { 3. One of the eigenmodes }\left\{\phi_{1}, \phi_{2}\right\} \text { is of parity class } 1, \\
\text { while the other is of parity class } 2 \text { as given in Table I. }
\end{array}\right.
$$

$$
\phi=\sum_{i=1}^{\infty} y_{i} \phi_{i}
$$

with sufficiently small initial data. Then, in the proof of the main theorem, we show that the dynamics of the system close to $R=R_{c}$ and $L=L_{c}$ is governed by the equations

$$
\begin{aligned}
& \frac{d y_{1}}{d t}=\beta_{1} y_{1}+y_{1}\left(a_{11} y_{1}^{2}+a_{13} y_{2}^{2}\right)+o(3), \\
& \frac{d y_{2}}{d t}=\beta_{2} y_{2}+y_{2}\left(a_{22} y_{1}^{2}+a_{24} y_{2}^{2}\right)+o(3) .
\end{aligned}
$$

Here, $\beta_{i}$ is the eigenvalue corresponding to mode $i$, and $o(3)$ denotes

$$
o(3)=o\left(\left|\left(y_{1}, y_{2}\right)\right|^{3}\right)+o\left(\left|\left(y_{1}, y_{2}\right)\right|^{3} \max _{i=1,2}|\beta(R)|\right) \text {. }
$$

Let us define

$$
D_{1}=a_{22} \beta_{1}-a_{11} \beta_{2}, \quad D_{2}=a_{13} \beta_{2}-a_{24} \beta_{1}, \quad D_{3}=a_{11} a_{24}-a_{13} a_{22}
$$

To state our main theorems, we assume the following non-degeneracy conditions

$$
a_{11} \neq 0, a_{24} \neq 0, D_{1} \neq 0, D_{2} \neq 0, D_{3} \neq 0
$$

Finally, let us define the following.

$$
\begin{array}{rlrl}
\varphi_{i} & =(-1)^{i} \phi_{1}, & i=1,2, \\
\varphi_{i}=(-1)^{i} \phi_{2}, & i=3,4, \\
\varphi_{i}=c_{i} \phi_{1}+d_{i} \phi_{2}, & i=5,6,7,8,
\end{array}
$$

where $c_{5}=c_{6}=-c_{7}=-c_{8}$ and $d_{5}=-d_{6}=d_{7}=-d_{8}$.

Thus, $\varphi_{1}$ and $\varphi_{2}$ are modes with wave number $k, \varphi_{3}$ and $\varphi_{4}$ are modes with wave number $k+1$, and $\varphi_{5}, \ldots, \varphi_{8}$ are mixed modes meaning that they are superpositions of the modes with wave numbers $k$ and $k+1$.

\section{Theorem 4.1}

Under the assumptions (19) and (22), there is an attractor $\Sigma_{R}$ bifurcating as $R$ crosses $R_{c}$, which is homeomorphic to the circle $S^{1}$ when $L$ is sufficiently close to a critical length scale $L_{c}$. Moreover, $\Sigma_{R}$ consists of steady states and their connecting heteroclinic orbits. Let $n$ ( $\Sigma_{R}$ ) denote the number of steady states on $\Sigma_{R}, \mathcal{S}$ denote the stable steady states, and $\mathcal{U}$ denote the unstable steady states on $\Sigma_{R}$ up to topological equivalency. Then, we have the following characterization of $\Sigma_{R}$, which is also illustrated in Figure 4.

(i) If $D_{1}<0, D_{2}<0, D_{3}<0, n\left(\Sigma_{R}\right)=8, \mathcal{S}=\left\{\varphi_{i} \mid i=1,2,3,4\right\}, \mathcal{U}=\left\{\varphi_{i} \mid i=5,6,7,8\right\}$.

(ii) If $D_{1}>0, D_{2}>0, D_{3}>0, n\left(\Sigma_{R}\right)=8, \mathcal{S}=\left\{\varphi_{i} \mid i=5,6,7,8\right\}, \mathcal{U}=\left\{\varphi_{i} \mid i=1,2,3,4\right\}$.

(iii) If $D_{1}<0, D_{2}>0, n\left(\Sigma_{R}\right)=4, \mathcal{S}=\left\{\varphi_{i} \mid i=1,2\right\}, \mathcal{U}=\left\{\varphi_{i} \mid i=3,4\right\}$.

(iv) If $D_{1}>0, D_{2}<0, n\left(\Sigma_{R}\right)=4, \mathcal{S}=\left\{\varphi_{i} \mid i=3,4\right\}, \mathcal{U}=\left\{\varphi_{i} \mid i=1,2\right\}$. 


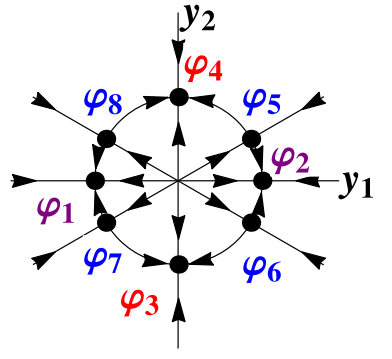

(a) $D_{1}<0, D_{2}<0, D_{3}<0$

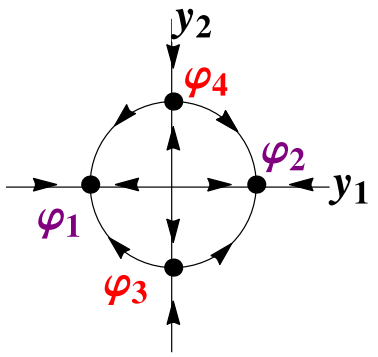

(c) $D_{1}<0, D_{2}>0$

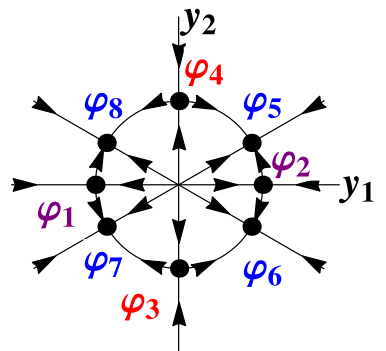

(b) $D_{1}>0, D_{2}>0, D_{3}>0$

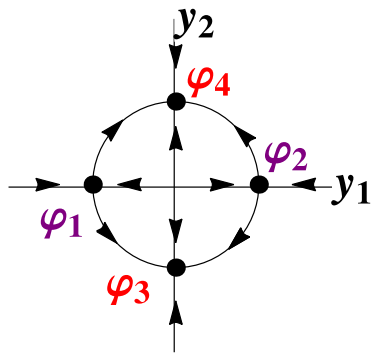

(d) $D_{1}>0, D_{2}<0$

Figure 4. Transition scenarios.

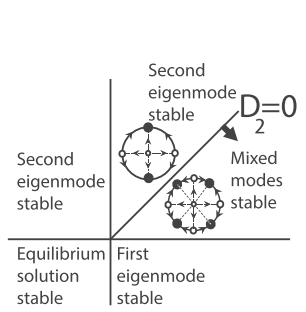

(a) $a_{22}>0$

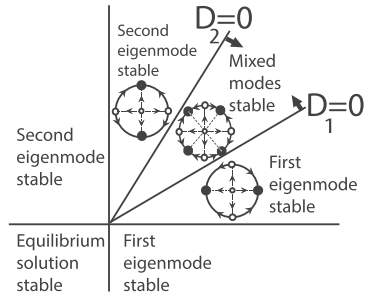

(b) $a_{22}<0, D_{3}>0$

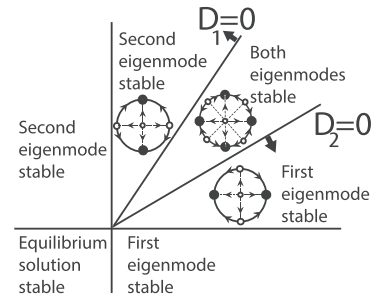

(c) $a_{22}<0, D_{3}<0$

Figure 5. The transition scenarios in $\beta_{1}-\beta_{2}$ plane. In the previous cases, we assume that $a_{13}<0$, which is due to our numerical observations. The arrows on the lines $D_{1}=0, D_{2}=0$ indicate in which directions $D_{1}$ and $D_{2}$ increase. First and second eigenmodes correspond to the eigenmodes with wave number $k$ and $k+1$, respectively.

According to Theorem 4.1, the structure of the attractor depends on $D_{1}, D_{2}$, and $D_{3}$, which in turn depends on the coefficients of the reduced equations. By (21), $D_{3}$ has a definite sign whereas $D_{1}$ and $D_{2}$ vanish at the criticality $\beta_{1}=\beta_{2}=0$. In the proof of Theorem 4.1, we analytically prove that the coefficients $a_{11}$ and $a_{24}$ are negative. Our numerical computations indicate that $a_{13}$ is also always negative. We observed that $a_{22}$ and $D_{3}$ can be both positive and negative.

That gives three possible cases depending on the signs of $a_{22}$ and $D_{3}$. In Figure 5, we classify these cases in a small neighborhood of $\beta_{1}=\beta_{2}=0$ in the $\beta_{1}-\beta_{2}$ plane according to our main theorem and the following observations.

- If $a_{22}>0$, then $D_{3}>0, D_{1}>0$, but $D_{2}$ changes sign in the first quadrant.

- If $a_{22}<0$ and $D_{3}>0$, then $D_{1}$ and $D_{2}$ change sign in the first quadrant. Moreover, the case where both $D_{1}<0$ and $D_{2}<0$ is not possible.

- If $a_{22}<0$ and $D_{3}<0$, then again $D_{1}$ and $D_{2}$ change sign in the first quadrant. This time the case where both $D_{1}>0$ and $D_{2}>0$ is not possible.

\section{Proof of the main theorem}

We will give the proof in several steps.

Step 1. The reduced equations. When there are two critical modes $\phi_{1}, \phi_{2}$, the center manifold is a 2D manifold embedded in the infinite dimensional space. We denote the center manifold function by

$$
\Phi=y_{1}^{2} \Phi_{1}+y_{1} y_{2} \Phi_{2}+y_{2}^{2} \Phi_{3}+o\left(y^{2}\right), \quad \Phi_{i}=\left(U_{i}, W_{i}, \Theta_{i}\right) .
$$


To study the dynamics on the center manifold, we plug in

$$
\phi=y_{1} \phi_{1}+y_{2} \phi_{2}+y_{1}^{2} \Phi_{1}+y_{1} y_{2} \Phi_{2}+y_{2}^{2} \Phi_{3}+o(2),
$$

into (5), take the inner product with $\phi_{1}, \phi_{2}$, and use the orthogonality of the eigenvectors, thanks to the self-adjointness of the linear operator. The reduced equations read

$$
\frac{d y_{i}}{d t}=\beta_{i}(R) y_{i}+\frac{1}{\left(\phi_{i}, \phi_{i}\right)}\left(G(\phi), \phi_{i}\right), \quad i=1,2
$$

We normalize the first two eigenfunctions so that

$$
\left(\phi_{1}, \phi_{1}\right)=\left(\phi_{2}, \phi_{2}\right)=1 \text {. }
$$

Now if we expand the nonlinear terms in (25), we obtain

$$
\begin{aligned}
& \frac{d y_{1}}{d t}=\beta_{1} y_{1}+\left(a_{11} y_{1}^{3}+a_{12} y_{1}^{2} y_{2}+a_{13} y_{1} y_{2}^{2}+a_{14} y_{2}^{3}\right)+o(3), \\
& \frac{d y_{2}}{d t}=\beta_{2} y_{2}+\left(a_{21} y_{1}^{3}+a_{22} y_{1}^{2} y_{2}+a_{23} y_{1} y_{2}^{2}+a_{24} y_{2}^{3}\right)+o(3),
\end{aligned}
$$

where

$$
\begin{array}{ll}
a_{k 1}=G_{s}\left(\phi_{1}, \Phi_{1}, \phi_{k}\right), & a_{k 2}=G_{s}\left(\phi_{1}, \Phi_{2}, \phi_{k}\right)+G_{s}\left(\phi_{2}, \Phi_{1}, \phi_{k}\right), \\
a_{k 4}=G_{s}\left(\phi_{2}, \Phi_{3}, \phi_{k}\right), & a_{k 3}=G_{s}\left(\phi_{1}, \Phi_{3}, \phi_{k}\right)+G_{s}\left(\phi_{2}, \Phi_{2}, \phi_{k}\right) .
\end{array}
$$

Step 2. Parities of the center manifold functions. To compute the center manifold approximation, we will use the following formula, which was introduced by Ma and Wang [15].

Here,

$$
\begin{aligned}
& -\mathcal{L}_{R} \Phi_{1}=P_{2} G\left(\phi_{1}, \phi_{1}\right), \\
& -\mathcal{L}_{R} \Phi_{2}=P_{2}\left[G\left(\phi_{1}, \phi_{2}\right)+G\left(\phi_{2}, \phi_{1}\right)\right], \\
& -\mathcal{L}_{R} \Phi_{3}=P_{2} G\left(\phi_{2}, \phi_{2}\right) .
\end{aligned}
$$

$$
\begin{array}{ll}
P_{2}: H \rightarrow E_{2}, & \mathcal{L}_{R}=\left.L_{R}\right|_{E_{2}}: E_{2} \rightarrow \bar{E}_{2}, \\
E_{1}=\operatorname{span}\left\{\phi_{1}, \phi_{2}\right\}, & E_{2}=E_{1}^{\perp} .
\end{array}
$$

Let $\mathcal{X}=\{f \in C(\Omega) \mid f(-x, z)= \pm f(x, z)$ and $f(x,-z)= \pm f(x, z)\}$, and let $s: \mathcal{X} \rightarrow\{ \pm 1\}^{2}$ denote the parity function:

$$
s(f)=\left(s_{X}(f), s_{Z}(f)\right),
$$

where

$$
s_{x}(f)= \pm 1 \quad \text { if } f(-x, z)= \pm f(x, z), \quad s_{z}(f)= \pm 1 \quad \text { if } f(x,-z)= \pm f(x, z) .
$$

Let us define for $\phi_{i}=\left(u_{i}, w_{i}, \theta_{i}\right), i=1,2$, the following.

$$
G\left(\phi_{i}, \phi_{j}\right)=\left[\begin{array}{l}
g_{1}\left(\phi_{i}, \phi_{j}\right) \\
g_{2}\left(\phi_{i}, \phi_{j}\right) \\
g_{3}\left(\phi_{i}, \phi_{j}\right)
\end{array}\right]=\left[\begin{array}{c}
-u_{i} \frac{\partial u_{j}}{\partial x}-w_{i} \frac{\partial u_{j}}{\partial z} \\
-u_{i} \frac{\partial w_{j}}{\partial x}-w_{i} \frac{\partial w_{j}}{\partial z} \\
-u_{i} \frac{\partial \theta_{j}}{\partial x}-w_{i} \frac{\partial \theta_{j}}{\partial z}
\end{array}\right] .
$$

The following lemma can be proven using the basic properties of parities.

Lemma 5.1

If $\phi_{i}=\left(u_{i}, v_{i}, \theta_{i}\right) \in \mathcal{X}^{3} \cap H_{1}, i=1,2$, then for $i, j, k=1,2$,

1. $-s\left(g_{1}\left(\phi_{i}, \phi_{j}\right)\right)=s\left(g_{2}\left(\phi_{i}, \phi_{j}\right)\right)=s\left(g_{3}\left(\phi_{i}, \phi_{j}\right)\right)=\left(s_{x}\left(w_{i} w_{j}\right),-s_{z}\left(w_{i} w_{j}\right)\right)$.

2. $s\left(g_{k}\left(\phi_{i}, \phi_{j}\right)\right)=s\left(g_{k}\left(\phi_{j}, \phi_{i}\right)\right)$.

Hereafter, without loss of generality, we will assume that

$$
\phi_{1} \text { is of parity class } 1 \text { and } \phi_{2} \text { is of parity class } 2,
$$

which are as given in Table I.

Using Lemma 5.1, we can prove

Lemma 5.2

Under the assumption (30),

$$
s\left(g_{2}\left(\phi_{1}, \phi_{1}\right)\right)=s\left(g_{2}\left(\phi_{2}, \phi_{2}\right)\right)=(1,-1), \quad s\left(g_{2}\left(\phi_{1}, \phi_{2}\right)\right)=(-1,-1) \text {. }
$$

Lemma 5.3

Under the assumption (30), $P_{2} G\left(\phi_{i}, \phi_{j}\right)=G\left(\phi_{i}, \phi_{j}\right)$ for $i, j=1,2$. 


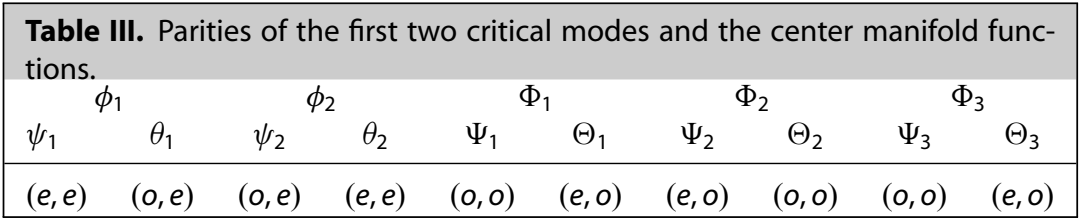

Proof

Note that $P_{2} G\left(\phi_{i}, \phi_{j}\right)=G\left(\phi_{i}, \phi_{j}\right)$ if $\left(G\left(\phi_{i}, \phi_{j}\right), \phi_{k}\right)=0$ for $i, j, k=1,2$. Now,

$$
\left(G\left(\phi_{i}, \phi_{j}\right), \phi_{k}\right)=\int_{\Omega}\left(g_{1}\left(\phi_{i}, \phi_{j}\right) u_{k}+g_{2}\left(\phi_{i}, \phi_{j}\right) w_{k}+g_{3}\left(\phi_{i}, \phi_{j}\right) \theta_{k}\right) d x d z
$$

By Lemma 5.1 and Lemma 5.2, $g_{1}\left(\phi_{i}, \phi_{j}\right)$ is even in the $z$-direction while $g_{2}\left(\phi_{i}, \phi_{j}\right)$ and $g_{3}\left(\phi_{i}, \phi_{j}\right)$ are odd in the $z$-direction. Because $u_{k}$ is odd and $w_{k}$ and $\theta_{k}$ are even in the $z$-direction, the integral in (31) must vanish over $\Omega$.

Thus, by Lemma 5.3 and equation (28), $\Phi_{i}=\left(U_{i}, W_{i}, \Theta_{i}\right),(i=1,2,3)$ are solutions of

$$
\begin{aligned}
& -\mathcal{L}_{R} \Phi_{1}=G\left(\phi_{1}, \phi_{1}\right), \\
& -\mathcal{L}_{R} \Phi_{2}=G\left(\phi_{1}, \phi_{2}\right)+G\left(\phi_{2}, \phi_{1}\right), \\
& -\mathcal{L}_{R} \Phi_{3}=G\left(\phi_{2}, \phi_{2}\right) .
\end{aligned}
$$

Using the stream function $\Psi_{z}=U, \Psi_{x}=-W$, one can eliminate the pressure from these equations to obtain

$$
\begin{gathered}
\operatorname{Pr} \Delta^{2} \Psi-\sqrt{R} \sqrt{\operatorname{Pr} \frac{\partial \Theta}{\partial x}}=h_{1}:=-\frac{\partial g_{1}}{\partial z}+\frac{\partial g_{2}}{\partial x}, \\
-\sqrt{R} \sqrt{\operatorname{Pr} \frac{\partial \Psi}{\partial x}+\Delta \theta}=h_{2}:=-g_{3} \\
\Psi=\frac{\partial \Psi}{\partial n}=\Theta=0 \text { on } \partial \Omega .
\end{gathered}
$$

Lemma 5.4

Under the assumption (30), the center manifold functions have the parity as given in Table III.

Proof

We can eliminate $\Theta$ from the first equation of (33) to obtain

$$
\begin{aligned}
\operatorname{Pr} \Delta^{3} \Psi-R \operatorname{Pr} \frac{\partial^{2} \Psi}{\partial x^{2}} & =\Delta h_{1}+\sqrt{R} \sqrt{\operatorname{Pr} \frac{\partial h_{2}}{\partial x}} \\
\Delta \Theta & =h_{2}+\sqrt{R} \sqrt{\operatorname{Pr}} \frac{\partial \Psi}{\partial x} .
\end{aligned}
$$

Now using Lemma 5.1 and Lemma 5.2, we see that $s(\Psi)=\left(-s_{x}\left(g_{2}\right), s_{z}\left(g_{2}\right)\right)$ and $s(\Theta)=s\left(g_{2}\right)$.

Using Table III, we find that the integrands in $a_{12}, a_{14}, a_{21}, a_{23}$ are all odd functions of $z$, and hence, we have the following.

Lemma 5.5

Under the assumption (30), in (27), we have

$$
a_{12}=a_{14}=a_{21}=a_{23}=0 .
$$

As a result of Lemma 5.5, we obtain the reduced equations (20).

Step 3. The attractor bifurcation. Now, we will prove that the bifurcated attractor is homeomorphic to $S^{1}$. For this, we will need the following result.

Theorem 5.6 (Ma and Wang [15])

Let $v$ be a $2 \mathrm{D} C^{r}(r \geq 1)$ vector field given by

$$
v_{\lambda}(x)=\beta(\lambda) x-h(x, \lambda),
$$

for $x \in \mathbb{R}^{2}$. Here, $\beta(\lambda)$ is a continuous function of $\lambda$ satisfying $\beta(\lambda) \underset{<}{\gtrless} 0$ for $\lambda \underset{<}{\gtrless} \lambda_{0}$ and

$$
h(x, \lambda)=h_{k}(x, \lambda)+o\left(|x|^{k}\right), \quad C_{1}|x|^{k+1} \leq\left(h_{k}(x, \lambda), x\right),
$$

for some odd integer $k \geq 3$ where $h_{k}(\cdot, \lambda)$ is a $k$-multilinear field, and $C_{1}>0$ is some constant. Then, the system

$$
d x / d t=v_{\lambda}(x), \quad x \in \mathbb{R},
$$

bifurcates from $(x, \lambda)=\left(0, \lambda_{0}\right)$ to an attractor $\Sigma_{\lambda}$, which is homeomorphic to $S^{1}$, for $\lambda_{0}<\lambda<\lambda_{0}+\epsilon$, for some $\epsilon>0$. Moreover, either (i) $\Sigma_{\lambda}$ is a periodic orbit, or (ii) $\Sigma_{\lambda}$ consists of an infinite number of singular points, or (iii) $\Sigma_{\lambda}$ contains at most 2(k+1) singular points, consisting of $2 N$ saddle points, $2 N$ stable node points, and $n(\leq 2(k+1)-4 N)$ singular points with index zero. 
Now let

$$
h\left(y_{1}, y_{2}\right)=\left\{y_{1}\left(a_{11} y_{1}^{2}+a_{13} y_{2}^{2}\right), y_{2}\left(a_{22} y_{1}^{2}+a_{24} y_{2}^{2}\right)\right\}^{T}
$$

Lemma 5.7

Assume that $\Phi_{i} \neq 0$ for $i=1,2,3$. Then for any $y=\left(y_{1}, y_{2}\right)$,

$$
(h(y), y)=a_{11} y_{1}^{4}+\left(a_{13}+a_{22}\right) y_{1}^{2} y_{2}^{2}+a_{24} y_{2}^{4} \leq C|y|^{4},
$$

where $C<0$.

Proof

$$
\begin{aligned}
a_{11} & =G_{s}\left(\phi_{1}, \Phi_{1}, \phi_{1}\right)=G\left(\phi_{1}, \Phi_{1}, \phi_{1}\right)+G\left(\Phi_{1}, \phi_{1}, \phi_{1}\right) \\
& =G\left(\phi_{1}, \Phi_{1}, \phi_{1}\right)=-G\left(\phi_{1}, \phi_{1}, \Phi_{1}\right)=\left(\mathcal{L}_{R} \Phi_{1}, \Phi_{1}\right) .
\end{aligned}
$$

Here, we used (32) and the following properties of the Navier-Stokes nonlinearity

$$
\text { (i) } G\left(\phi, \tilde{\phi}, \phi^{*}\right)=G\left(\phi, \phi^{*}, \tilde{\phi}\right), \quad \text { (ii) } G(\phi, \tilde{\phi}, \tilde{\phi})=0,
$$

and $-\mathcal{L}_{R} \Phi_{1}=G\left(\phi_{1}, \phi_{1}\right)$, which is due to (32).

If we write

then for $j=1,2,3$,

$$
\Phi_{j}=\sum_{k=3}^{\infty} c_{j, k} \phi_{k}, \quad j=1,2,3
$$

$$
\left(\mathcal{L}_{R} \Phi_{j}, \Phi_{j}\right)=\sum_{k=3}^{\infty} c_{j, k}^{2} \beta_{k}\left\|\phi_{k}\right\|^{2}<0 .
$$

Because $\beta_{k}<0$ for $k \geq 3$ and by assumption, there exists $k \geq 3$ such that $c_{1, k} \neq 0$. In particular, $a_{11}<0$. As in (37), we can show that

$$
a_{24}=G_{s}\left(\phi_{2}, \Phi_{3}, \phi_{2}\right)=\left(\mathcal{L}_{R} \Phi_{3}, \Phi_{3}\right)<0 .
$$

Now if $a_{13}+a_{22}<0$, then it is easy to prove (36). Assume otherwise. Using (38) and (32), we can write

$$
\begin{aligned}
a_{13} & =G_{s}\left(\phi_{1}, \Phi_{3}, \phi_{1}\right)+G_{s}\left(\phi_{2}, \Phi_{2}, \phi_{1}\right) \\
& =G\left(\phi_{1}, \Phi_{3}, \phi_{1}\right)+G\left(\Phi_{3}, \phi_{1}, \phi_{1}\right)+G_{s}\left(\phi_{2}, \Phi_{2}, \phi_{1}\right) \\
& =-\left(G\left(\phi_{1}\right), \Phi_{3}\right)+G_{s}\left(\phi_{2}, \Phi_{2}, \phi_{1}\right) \\
& =\left(\mathcal{L}_{R} \Phi_{1}, \Phi_{3}\right)+G_{s}\left(\phi_{2}, \Phi_{2}, \phi_{1}\right) .
\end{aligned}
$$

A similar computation shows

$$
a_{22}=\left(\mathcal{L}_{R} \Phi_{3}, \Phi_{1}\right)+G_{s}\left(\phi_{1}, \Phi_{2}, \phi_{2}\right)
$$

Let us define

$$
\alpha=G_{s}\left(\phi_{1}, \Phi_{2}, \phi_{2}\right)+G_{s}\left(\phi_{2}, \Phi_{2}, \phi_{1}\right) .
$$

By (38) and (32),

$$
\alpha=-\left(G\left(\phi_{1}, \phi_{2}\right)+G\left(\phi_{2}, \phi_{1}\right), \Phi_{2}\right)=\left(\mathcal{L}_{R} \Phi_{2}, \Phi_{2}\right) .
$$

Note that $\alpha<0$ by (39). By using Cauchy-Schwarz inequality and the orthogonality of the eigenfunctions,

$$
\begin{aligned}
\left(\mathcal{L}_{R} \Phi_{1}, \Phi_{3}\right) & =\sum_{k=3}^{\infty} \beta_{k} c_{1, k} c_{3, k}\left\|\phi_{k}\right\|^{2} \\
& \leq\left(\sum_{k=3}^{\infty}-\beta_{k} c_{1, k}^{2}\left\|\phi_{k}\right\|^{2}\right)^{1 / 2}\left(\sum_{k=3}^{\infty}-\beta_{k} c_{3, k}^{2}\left\|\phi_{k}\right\|^{2}\right)^{1 / 2} \\
& =\sqrt{a_{11} a_{24}} .
\end{aligned}
$$

Because $\left(\mathcal{L}_{R} \Phi_{3}, \Phi_{1}\right)=\left(\mathcal{L}_{R} \Phi_{1}, \Phi_{3}\right)$, we have by (40)-(44),

$$
a_{13}+a_{22}<2 \sqrt{a_{11} a_{24}}+\alpha
$$

where $\alpha<0$ is given by (42). Thus, there exists $0<\epsilon_{1}<-a_{11}, 0<\epsilon_{2}<-a_{24}$ such that

$$
a_{13}+a_{22}<2 \sqrt{a_{11} a_{24}}+\alpha<2 \sqrt{\left(a_{11}+\epsilon_{1}\right)\left(a_{24}+\epsilon_{2}\right)} \text {. }
$$

Because $2 a b<a^{2}+b^{2}$, we have

$$
2 \sqrt{\left(a_{11}+\epsilon_{1}\right)\left(a_{24}+\epsilon_{2}\right)} y_{1}^{2} y_{2}^{2} \leq-\left(a_{11}+\epsilon_{1}\right) y_{1}^{4}-\left(a_{24}+\epsilon_{2}\right) y_{2}^{4}
$$


Now, let $C=\max \left\{-\epsilon_{1},-\epsilon_{2}\right\}$. Then, $C<0$, and we have

$$
(h(y), y) \leq a_{11} y_{1}^{4}+\left(a_{13}+a_{22}\right) y_{1}^{2} y_{2}^{2}+a_{24} y_{2}^{4} \leq C\left(x^{2}+y^{2}\right)^{2} .
$$

That finishes the proof.

Thus, by Theorem 5.6 and Lemma $5.7, \Sigma_{R}$ is homeomorphic to $S^{1}$. Now we will describe the details of its structure by determining the bifurcated steady states and their stabilities.

Step 4. The steady states and their stabilities. The possible equilibrium solutions of the truncated equations of (20) are as follows.

$$
R_{1}=\left(\sqrt{\frac{\beta_{1}}{-a_{11}}}, 0\right), R_{2}=\left(0, \sqrt{\frac{\beta_{2}}{-a_{24}}}\right), M=\left(\sqrt{\frac{D_{2}}{D_{3}}}, \sqrt{\frac{D_{1}}{D_{3}}}\right),
$$

where $D_{1}, D_{2}$, and $D_{3}$ are given by (21).

Because of the invariance of the equations (20) with respect to $(x, y) \rightarrow(-x, y)$ and $(x, y) \rightarrow(x,-y)$, we only consider the positive solutions when writing (45).

The eigenvalues $\lambda_{1}, \lambda_{2}$ of the truncated vector field at the steady states $R_{1}, R_{2}$ are

$$
\lambda_{1}^{R_{1}}=-2 \beta_{1}, \lambda_{2}^{R_{1}}=-D_{1} / a_{11}, \lambda_{1}^{R_{2}}=-2 \beta_{2}, \lambda_{2}^{R_{2}}=-D_{2} / a_{24}
$$

Note that $R_{i}$ is always bifurcated for $\beta_{i}>0, i=1,2$. Moreover, $R_{i}$ is a stable steady state for $\beta_{i}>0$ if $D_{i}<0$ for $i=1,2$. The trace $\operatorname{Tr}$ and the determinant $D e t$ of the Jacobian matrix of the truncated vector field at the mixed states $M$ are

$$
\operatorname{Tr}=\frac{2}{D_{3}}\left(a_{24} D_{1}+a_{11} D_{2}\right), \quad \text { Det }=\frac{4}{D_{3}} D_{1} D_{2} .
$$

Notice that the steady states $M$ are bifurcated only when $D_{1}, D_{2}, D_{3}$ have the same sign. Because $a_{11}$ and $a_{24}$ are both negative as shown in Lemma 5.7, according to trace-determinant plane analysis, they are saddles if $D_{1}<0, D_{2}<0, D_{3}<0$ and are stable if $D_{1}>0, D_{2}>0$, $D_{3}>0$.

Finally, only the four cases stated in our main theorem can occur. To see this, note that according to Theorem 5.6 and (45)-(46), the case $D_{1}<0, D_{2}<0, D_{3}>0$ is not possible because that would lead to only four steady states on the attractor, which are all stable. Similarly, the case $D_{1}>0, D_{2}>0, D_{3}<0$ is not possible either, which would lead to four steady states, which are all unstable.

\section{Numerical approximation of the coefficients of the reduced equations}

To compute the coefficients of the reduced equations (20), we fix $L, \operatorname{Pr}$, and $R$ to compute all the eigenvalues $\beta_{i}^{N}$ and the corresponding eigenvectors of (16).

Numerical computation of the center manifold functions. Now we will numerically approximate $\Phi_{1}, \Phi_{2}$, and $\Phi_{3}$, which are the solutions of the equations (28). We will illustrate the method to approximate $\Phi_{1}$ because $\Phi_{2}, \Phi_{3}$ can be approximated similarly. To determine $\Phi_{1}$, we have to find its stream function $\Psi$ and its temperature function $\Theta$, which are determined by the equations (33).

Because we do not have $h_{1}$ and $h_{2}$ in (33) exactly, we approximate them by $h_{1}^{N}, h_{2}^{N}$ as that shown later.

$$
\begin{array}{ll}
h_{1}^{N}=-\frac{\partial g_{1}^{N}}{\partial z}+\frac{\partial g_{2}^{N}}{\partial x}, & h_{2}^{N}=\psi_{1, z}^{N} \theta_{1, x}^{N}-\psi_{1, x}^{N} \theta_{1, z^{\prime}}^{N} \\
g_{1}^{N}=-\psi_{1, z}^{N} \psi_{1, x z}^{N}+\psi_{1, x}^{N} \psi_{1, z z^{\prime}}^{N}, & g_{2}^{N}=-\psi_{1, z}^{N} \psi_{1, x x}^{N}+\psi_{1, x}^{N} \psi_{1, x z}^{N} .
\end{array}
$$

Here, $\left(\psi_{1}^{N}, \theta_{1}^{N}\right)$ is the first critical eigenfunction of the discrete problem (16).

$$
\left\{\psi_{1}^{N}, \theta_{1}^{N}\right\}=\sum_{m=0}^{N_{x}-1} \sum_{n=0}^{N_{z}-1}\left\{\tilde{\psi}_{1, m n}^{N} e_{m}(x) e_{n}(z), \tilde{\theta}_{1, m n}^{N} f_{m}(x) f_{n}(z)\right\} .
$$

The Legendre-Galerkin approximation of the problem (33). As in the linear eigenvalue problem, we discretize the equations (33) using the generalized Jacobi polynomials (11)-(12).

$$
\left\{\Psi^{N}, \Theta^{N}\right\}=\sum_{m=0}^{N_{x}-1} \sum_{n=0}^{N_{z}-1}\left\{\tilde{\Psi}_{m n}^{N} e_{m}(x) e_{n}(z), \tilde{\Theta}_{m n}^{N} f_{m}(x) f_{n}(z)\right\} .
$$

We plug in $\Psi^{N}, \Theta^{N}, h_{1}^{N}, h_{2}^{N}$ for $\Psi, \Theta, h_{1}, h_{2}$ in (33) and multiply the resulting equations by Jacobi polynomials $e_{j}(x) e_{k}(z), f_{j}(x) f_{k}(z)$ and integrate over $-1 \leq x \leq 1,-1 \leq z \leq 1$ to reduce (33) to the following finite dimensional linear equation

$$
\left(B^{N}-\sqrt{R} C^{N}\right) \bar{x}=\bar{b} .
$$


Here, $B^{N}$ and $C^{N}$ are given by (17) and

$$
\bar{x}=\left[\operatorname{vec}\left(\tilde{\Psi}^{N}\right) \operatorname{vec}\left(\tilde{\Theta}^{N}\right)\right]_{N^{2} \times 1}^{T}, \quad \bar{b}=\left[\operatorname{vec}\left(B_{1}\right) \operatorname{vec}\left(B_{2}\right)\right]^{T} .
$$

For $0 \leq j \leq N_{x}-1,0 \leq k \leq N_{z}-1$,

$$
\begin{aligned}
\left(B_{1}\right)_{j k} & =\int_{-1}^{1} \int_{-1}^{1} h_{1}^{N}(x, z) e_{j}(x) e_{k}(z) d x d z, \\
\left(B_{2}\right)_{j k} & =\int_{-1}^{1} \int_{-1}^{1} h_{2}^{N}(x, z) f_{j}(x) f_{k}(z) d x d z .
\end{aligned}
$$

Now, $e_{j}$ is a polynomial of degree $j+4$, and by (47) and (48), $h_{i}^{N}$ is a polynomial of degree at most $\left(2 N_{x}+6,2 N_{z}+6\right)$. Thus, the aforementioned integrands are of degree at most $\left(3 N_{x}+9,3 N_{z}+9\right)$. Because the Legendre-Gauss-Lobatto quadrature with $N+1$ quadrature points is exact for polynomials of degree less or equal than $2 N-1$, the integrals in (52) can be replaced by the following discrete inner products.

$$
\begin{aligned}
\left(B_{1}\right)_{j k} & =\sum_{m=0}^{\frac{3}{2} N_{x}+5} \sum_{n=0}^{\frac{3}{2} N_{z}+5} h_{1}^{N}\left(x_{m}, z_{n}\right) e_{j}\left(x_{m}\right) e_{k}\left(z_{n}\right) \omega_{m}^{x} \omega_{n^{\prime}}^{z} \\
\left(B_{2}\right)_{j k} & =\sum_{m=0}^{\frac{3}{2} N_{x}+5} \sum_{n=0}^{\frac{3}{2} N_{z}+5} h_{2}^{N}\left(x_{m}, z_{n}\right) f_{j}\left(x_{m}\right) f_{k}\left(z_{n}\right) \omega_{m}^{x} \omega_{n}^{z} .
\end{aligned}
$$

Here, $\left\{x_{j}, w_{j}^{x}\right\}_{j=0}^{\frac{3}{2} N_{x}+5}$ and $\left\{z_{j}, w_{j}^{z}\right\}_{j=0}^{\frac{3}{2} N_{z}+5}$ are the Legendre-Gauss-Lobatto points and weights in the $x$-direction and the $z$-direction.

Solution of (50). The solution $\bar{x}$ of (50) can be obtained by inverting the matrix $\left(B^{N}-\sqrt{R} C^{N}\right)$. But this matrix has a large condition number. Thus, we show a method to obtain the solution inverting the matrix $D^{N}$ given by (17), which has a much smaller condition number. For example, for $N_{x}=10, N_{z}=8$, the condition number of $\left(B^{N}-\sqrt{R} C^{N}\right)$ is $O\left(10^{16}\right)$ while the condition number of $D^{N}$ is $O\left(10^{8}\right)$.

Because $\Phi_{1} \in E_{2}=\operatorname{span}\left\{\phi_{1}, \phi_{2}\right\}^{\perp}$, we look for a solution of (50) in the form

$$
\bar{x}=\sum_{i=3}^{N} x_{i} \bar{x}_{i}
$$

where $\bar{x}_{i}$ are the eigenvectors of

$$
B^{N} \bar{x}_{i}-\sqrt{R} C^{N} \bar{x}_{i}=\beta_{i}(R) D^{N} \bar{x}_{i}
$$

If we multiply (50) by $\left(D^{N}\right)^{-1}$ and use (55), the left-hand side of (50) becomes

$$
\sum_{i=3}^{N} x_{i} \beta_{i}(R) \bar{x}_{i}=\left(D^{N}\right)^{-1}\left(B^{N}-\sqrt{R} C^{N}\right) \bar{x}=\left(D^{N}\right)^{-1} \bar{b}:=\bar{f} .
$$

We determine $\bar{f}$ from $D^{N} \bar{f}=\bar{b}$ using Gaussian elimination. Once again using Gaussian elimination, we can find the coefficients $f_{i}$ in the expansion

$$
\bar{f}=\sum_{i=1}^{N} f_{i} \bar{x}_{i}
$$

In (57), we see that $f_{1}=f_{2}=0$ is necessary for the existence of a solution of (50). From (56) and (57), one finds $x_{i}=f_{i} / \beta_{i}(R)$, $i=3,4, \ldots, N$. Thus, the Jacobi expansion coefficients in (49) of the center manifold are given by

$$
\operatorname{vec}\left(\tilde{\Psi}^{N}\right)=\sum_{i=3}^{N} \frac{f_{i}}{\beta_{i}(R)} \operatorname{vec}\left(\tilde{\psi}_{i}^{N}\right), \quad \operatorname{vec}\left(\tilde{\Theta}^{N}\right)=\sum_{i=3}^{N} \frac{f_{i}}{\beta_{i}(R)} \operatorname{vec}\left(\tilde{\theta}_{i}^{N}\right)
$$

Numerical computation of $a_{i j}$ in (27). We approximate $a_{11}$ by

$$
a_{11}^{N}=G_{s}\left(\phi_{1}^{N}, \Phi_{1}^{N}, \phi_{1}^{N}\right)
$$

The integrands in $G_{s}\left(\phi_{1}^{N}, \Phi_{1}^{N}, \phi_{1}^{N}\right)$ are polynomials of degree at most ( $\left.3 N_{x}+9,3 N_{z}+9\right)$. Thus, to replace the integrals in (58), one needs again $\left(\frac{3}{2} N_{x}+5, \frac{3}{2} N_{z}+5\right)$ quadrature points and nodes in the numerical inner product. The other coefficients $a_{i j}$ in (27) are approximated similarly. 


\begin{tabular}{|c|c|c|c|c|c|c|}
\hline \multirow[b]{2}{*}{$\operatorname{Pr}$} & \multicolumn{2}{|c|}{$L_{c}=1.5702$} & \multicolumn{2}{|c|}{$L_{c}=2.6611$} & \multicolumn{2}{|c|}{$L_{c}=3.7048$} \\
\hline & $a_{22} \times 10^{2}$ & $D_{3} \times 10^{5}$ & $a_{22} \times 10^{2}$ & $D_{3} \times 10^{5}$ & $a_{22} \times 10^{2}$ & $D_{3} \times 10^{5}$ \\
\hline 0.01 & 7.6492 & 2559.8276 & -23.7252 & 140.2894 & -20.5185 & -15.6696 \\
\hline 0.04 & 0.1313 & 93.3662 & -6.0784 & 3.6690 & -5.3093 & -1.4416 \\
\hline 0.05 & -0.4546 & 50.5609 & -5.0374 & 1.0455 & -4.4332 & -1.1525 \\
\hline 0.06 & -0.8740 & 29.9252 & -4.3902 & -0.2713 & -3.8966 & -1.0122 \\
\hline 0.14 & -2.3697 & 0.6458 & -3.0486 & -2.3788 & -2.8749 & -0.9899 \\
\hline 0.15 & -2.4662 & -0.0707 & -3.0218 & -2.4414 & -2.8641 & -1.0182 \\
\hline 0.71 & -3.2469 & -9.5827 & -2.8940 & -2.5437 & -2.8848 & -1.4316 \\
\hline 7 & -0.8551 & -0.8348 & -0.7484 & -0.1610 & -0.7444 & -0.0987 \\
\hline 100 & -0.0687 & -0.0055 & -0.0599 & -0.0010 & -0.0595 & -0.0006 \\
\hline 1000 & -0.0069 & -0.0001 & -0.0060 & -0.00001 & -0.0060 & -0.000006 \\
\hline
\end{tabular}

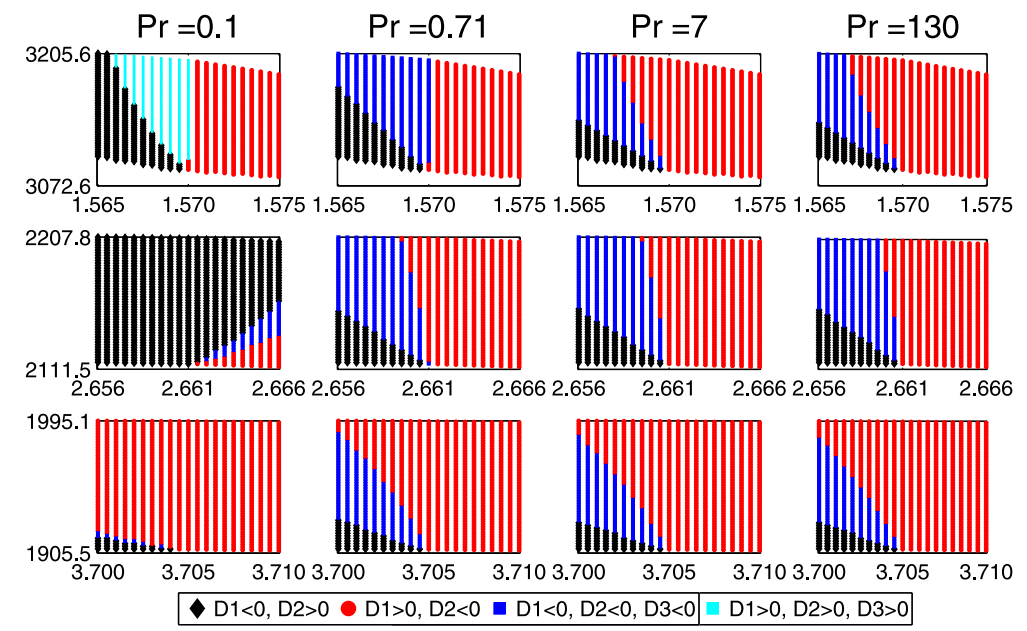

Figure 6. The signs of $D_{1}, D_{2}$, and $D_{3}$ in the $L-R$ plane. In each subfigure, the $x$ and $y$ axes denote the length scale $L$ and the Rayleigh number $R$, respectively. For each column, the Prandtl number is given earlier.

Remark 6.1

We observed that increasing $N_{x}$ and $N_{z}$ above $N_{x}=10+2 k$ and $N_{z}=8$ only changes $a_{i j}^{N}$ in the seventh digit when the first critical mode has $k$ rolls and the second critical mode has $k+1$ rolls in their stream functions.

\section{Numerical results and discussion}

We computed coefficients of the reduced equations for various $\operatorname{Pr}$ values ranging from 0.1 to $10^{3}$ at the first three critical length scales and at the critical Rayleigh numbers, which are given in Table II.

As proven in Theorem 4.1, the coefficients $a_{11}$ and $a_{24}$ are always negative. In our numerical calculations, we encountered that $a_{13}$ is also always negative. But the sign of $a_{22}$ and the sign of $D_{3}$ depend on $L$ and $\operatorname{Pr}$ and are given in Table IV.

For the first critical length scale $L_{c}=1.5702$, we found that $a_{22}$ and $D_{3}$ change sign from positive to negative between $0.04<$ $\operatorname{Pr}<0.05$ and $0.14<\operatorname{Pr}<0.15$, respectively. Thus, the transition is as described in Figure 5(a) for $\operatorname{Pr}<0.04$, as in Figure 5(b) for $0.05<\operatorname{Pr}<0.14$, and as in Figure 5(c) for $\operatorname{Pr}>0.15$. Thus, the mixed modes can be stable when $\operatorname{Pr}<0.14$, but only the pure modes are stable points of the attractor when $\operatorname{Pr}>0.15$.

For the second critical length scale $L_{c}=2.6611$, we always observed that $a_{22}<0$. However, $D_{3}$ changes sign between $0.05<\operatorname{Pr}<$ 0.06. Thus, the transition is as described in Figure 5(b) for $\operatorname{Pr}<0.05$ and as described in Figure 5 (c) for $\operatorname{Pr}>0.06$. In particular, the mixed modes can be stable when $\operatorname{Pr}<0.05$, but only the pure modes are stable steady states when $\operatorname{Pr}>0.06$.

For higher critical length scales (third and beyond), we found that $a_{22}<0$ and $D_{3}<0$ for the Prandtl numbers we considered. Thus, the transition is as described in Figure 5(c). For this length scale, either the critical Prandtl number that was observed for the first two critical length scales is now very close to zero, or it does not exist at all.

The previous analysis depends on the coefficients $a_{i j}$ of the reduced equations and predicts the transitions when both eigenvalues $\beta_{1}, \beta_{2}$ are close to zero. Now, we present an analysis depending on the direct computation of the numbers $D_{1}, D_{2}$ (both of which vanish when $\beta_{1}=\beta_{2}=0$ ), and $D_{3}$. We computed $D_{1}, D_{2}$, and $D_{3}$ for $L$ and $R$ values around (but not necessarily very close to) the criticality $(L, R)=\left(L_{c}, R_{c}\right)$ for the first three critical length scales and for Prandtl numbers $\operatorname{Pr}=0.1,0.71,7,130$. The results are shown in Figure 6. 
Although we might have omitted the smallness assumptions of $\left|L-L_{c}\right|$ and $\left|R-R_{c}\right|$ where our main theorem is valid, these figures help us predict the transitions in the $L-R$ plane. The results we obtain are as follows.

For $\operatorname{Pr}=0.71, \operatorname{Pr}=7, \operatorname{Pr}=130$, transitions are qualitatively the same in the $L-R$ plane. For $L>L_{c}$, the basic motionless state loses its stability to the eigenmode with wave number $k+1$ as the Rayleigh number crosses the first critical Rayleigh number and further increase of the Rayleigh number does not alter the stability of this steady state. This is in contrast to the situation $L<L_{c}$ where there is a transition of stabilities as the Rayleigh number is increased. Namely, as the Rayleigh number crosses the first critical Rayleigh number, the eigenmode with wave number $k$ becomes stable. As the Rayleigh number is further increased, both eigenmodes coexist as stable steady states and the initial conditions determine which one of these steady states will be realized. Finally, as the Rayleigh number is further increased, the eigenmode with wave number $k+1$ becomes stable.

The transition at $\operatorname{Pr}=0.1$ is essentially different than for those at $\operatorname{Pr}=0.71,7,130$. In particular, for the first critical length scale $L_{c}=1.5702$, for $L<L_{c}$, subsequently, mode with wave number $k$, mixed modes, and finally mode with wave number $k+1$ will be realized as the Rayleigh number is increased while for $L>L_{c}, k+1$ mode is the only stable steady state.

\section{Acknowledgements}

The work of Shen is partially supported by NSF-DMS-1217066 and DMS-1419053, and the work of Wang is supported in part by NSFDMS-1211218.

\section{References}

1. Davies-Jones RP. Thermal convection in an infinite channel with no-slip sidewalls. Journal of Fluid Mechanics 1970; 44(4):695-704.

2. Luijkx JM, Platten JK. On the onset of free convection in a rectangular channel. Journal of Non-Equilibrium Thermodynamics 1981; 6(3):141-158.

3. Kato Y, Fujimura K. Prediction of pattern selection due to an interaction between longitudinal rolls and transverse modes in a flow through a rectangular channel heated from below. Physical Review E 2000; 62(1):601-611.

4. Lee NY, Schultz WW, Boyd JP. Stability of fluid in a rectangular enclosure by spectral method. International Journal of Heat and Mass Transfer 1989; 32(3):513-520.

5. Mizushima J. Onset of the thermal convection in a finite two-dimensional box. Journal of the Physical Society of Japan 1995; 64(7):2420-2432.

6. Gelfgat AY. Different modes of Rayleigh-Bénard instability in two- and three-dimensional rectangular enclosures. Journal of Computational Physics 1999; 156(2):300-324.

7. Ma T, Wang S. Dynamic bifurcation and stability in the Rayleigh-Bénard convection. Communications in Mathematical Sciences 2 2004; 2:159-183.

8. Ma T, Wang S. Rayleigh-Bénard convection: dynamics and structure in the physical space. Communications in Mathematical Sciences 2007; 5(3):553574.

9. Sengul T, Wang S. Pattern formation in Rayleigh-Bénard convection. Communications in Mathematical Sciences 11 2013; 1:315-343.

10. Orszag SA. Accurate solution of the Orr-Sommerfeld stability equation. Journal of Fluid Mechanics 1971; 50(4):689-703.

11. Hill AA, Straughan B. A Legendre spectral element method for eigenvalues in hydrodynamic stability. Journal of computational and applied mathematics 2006; 193(1):363-381.

12. Gheorghiu Cl, Dragomirescu FI. Spectral methods in linear stability. Applications to thermal convection with variable gravity field. Applied Numerical Mathematics 2009; 59(6):1290-1302.

13. Ma T, Wang S. Phase Transition Dynamics. Springer-Verlag, 2013.

14. Foias C, Manley O, Temam R. Attractors for the Bénard problem: existence and physical bounds on their fractal dimension. Nonlinear Analysis 1987; 11(8):939-967.

15. Ma T, Wang S. Bifurcation Theory and Applications, Vol. 53. World Scientific Publishing: Singapore, 2005.

16. Hydon PE. Symmetry Methods for Differential Equations: A Beginner's Guide, Vol. 22. Cambridge University Press: Cambridge, UK, 2000.

17. Marques F, Lopez JM, Blackburn HM. Bifurcations in systems with $Z_{2}$ spatio-temporal and $\mathrm{O}(2)$ spatial symmetry. Physica D: Nonlinear Phenomena 2004; 189(3):247-276.

18. Shen J. Efficient spectral-Galerkin method I. Direct solvers of second- and fourth-order equations using Legendre polynomials. SIAM Journal on Scientific Computing 1994; 15(6):1489-1505.

19. Shen J, Tang T, Wang L. Spectral Methods: Algorithms, Analysis and Applications, Vol. 40. Springer Verlag, 2011.

20. Guo B, Shen J, Wang L. Optimal spectral-Galerkin methods using generalized Jacobi polynomials. Journal of Scientific Computing 2006; 27(1-3): 305-322.

21. Mizushima J, Nakamura T. Repulsion of eigenvalues in the Rayleigh-Bénard problem. Journal of the Physical Society of Japan 2002; 71:677. 